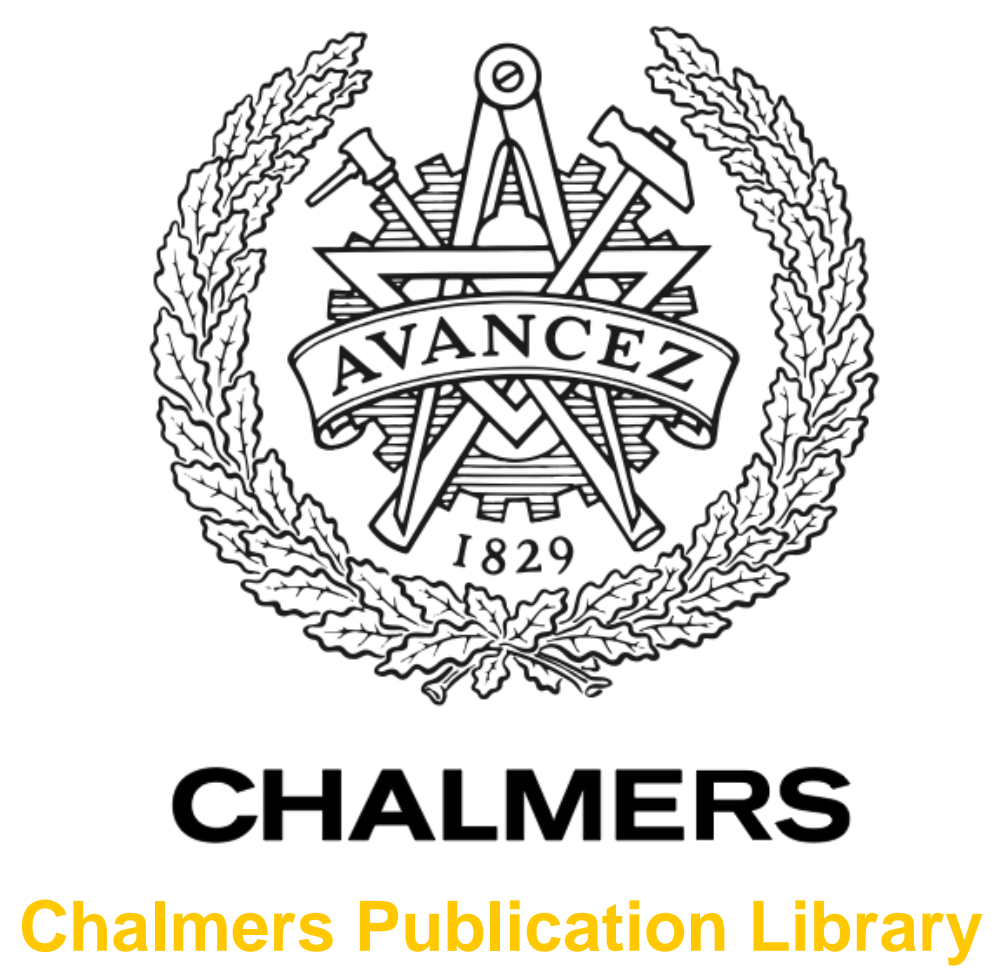

Optical response of a titanium-based cold-electron bolometer

This document has been downloaded from Chalmers Publication Library $(\mathrm{CPL})$. It is the author's version of a work that was accepted for publication in:

Superconductor Science \& Technology (ISSN: 0953-2048)

Citation for the published paper:

Otto, E. ; Tarasov, M. ; Grimes, P. (2013) "Optical response of a titanium-based coldelectron bolometer". Superconductor Science \& Technology, vol. 26(8),

http://dx.doi.org/10.1088/0953-2048/26/8/085020

Downloaded from: http://publications.lib.chalmers.se/publication/181459

Notice: Changes introduced as a result of publishing processes such as copy-editing and formatting may not be reflected in this document. For a definitive version of this work, please refer to the published source. Please note that access to the published version might require a subscription. 
Optical response of a titanium-based cold-electron bolometer

This article has been downloaded from IOPscience. Please scroll down to see the full text article.

2013 Supercond. Sci. Technol. 26085020

(http://iopscience.iop.org/0953-2048/26/8/085020)

View the table of contents for this issue, or go to the journal homepage for more

Download details:

IP Address: 129.16.137.136

The article was downloaded on 05/07/2013 at 13:25

Please note that terms and conditions apply. 


\title{
Optical response of a titanium-based cold-electron bolometer
}

\author{
Ernst Otto $^{1,2}$, Mikhail Tarasov ${ }^{2,3}$, Paul K Grimes ${ }^{1,4}$, \\ Artem Chekushkin ${ }^{2,3}$, Leonid S Kuzmin ${ }^{2}$ and Ghassan Yassin ${ }^{1}$ \\ ${ }^{1}$ Department of Physics, Oxford University, Oxford, UK \\ ${ }^{2}$ Chalmers University of Technology, Gothenburg, Sweden \\ ${ }^{3}$ Kotel'nikov Institute of Radio Engineering and Electronics of Russian Academy of Sciences, Moscow, \\ Russia \\ ${ }^{4}$ Smithsonian Astrophysical Observatory, Cambridge, MA, USA \\ E-mail: otto.mc2@gmail.com
}

Received 7 March 2013, in final form 3 June 2013

Published 4 July 2013

Online at stacks.iop.org/SUST/26/085020

\begin{abstract}
We present experimental results on the testing of cold-electron bolometer (CEB) detectors comprised of a thin Ti film absorber and two SIN junctions integrated with a planar antenna. The CEB performance was tested in a $\mathrm{He}^{3}$ sorption cryostat HELIOX-AC-V at bath temperatures of 280-305 mK. The optical response was measured using the hot/cold load method by flipping a $\mathrm{Cu}$ reflector opposite a blackbody surface inside a $3 \mathrm{~K}$ shield and using a thermal source with variable temperature. In the first experiment, the detector chip was mounted in an optical sample-holder whose aperture was switched towards or away from a blackbody source changing the incident radiation temperature from $3 \mathrm{~K}$ to $270 \mathrm{mK}$. As a result, we measured the optical response to a $3 \mathrm{~K} / 270 \mathrm{mK}$ radiation temperature change. The measured voltage response value for the detector integrated in a double-dipole antenna was $\Delta V_{\text {out }}=120 \mu \mathrm{V}$. This corresponds to a noise equivalent power of $\mathrm{NEP}=V_{n} /(\mathrm{d} V / \mathrm{d} P)=3.5 \times 10^{-17} \mathrm{~W} \mathrm{~Hz}^{-1 / 2}$, where $\mathrm{d} V / \mathrm{d} P$ is the voltage to power response obtained from the incoming power estimation based on the Planck formula.
\end{abstract}

(Some figures may appear in colour only in the online journal)

\section{Introduction}

There has been extensive activity in the past few years to develop a new generation of bolometric detectors for telescopes aiming to measure the CMB polarization and for far-infrared spectroscopy. One candidate technology is the cold-electron bolometer (CEB).

The CEB [1,2] is a millimeter-wave detector which is being developed for cosmology instruments. It has high sensitivity, high saturation power and very fast response, which are important features for applications in astronomy. A key advantage of the CEB is that it can easily be coupled to a planar antenna since it can be fabricated on a silicon substrate without the need of fragile membranes. Also the output impedance of the device can be varied between low or high dynamic resistance depending on the choice of readout. The CEB devices considered here consist of a normal metal absorber coupled to superconducting electrodes via SIN tunnel junctions at each end of the absorber. RF power from the antenna is coupled through the SIN tunnel junctions into the absorber, which excites electrons in the absorber, increasing the electron temperature over that of the phonons. The hottest electrons can tunnel through the junction oxide barrier, cooling the electron system, and giving a DC current that increases with the temperature of the electron system. Thus when RF power is coupled to the absorber, the electrons in the absorber are heated, and the DC tunnel current increases, providing a measure of the power incident on the device.

The electron cooling of the device increases the dynamic range of the system, thus substantially increasing the detector saturation power. This is to be contrasted with the operation of a transition edge sensor (TES) where the constant voltage biasing source heats the device in order to keep it near the 

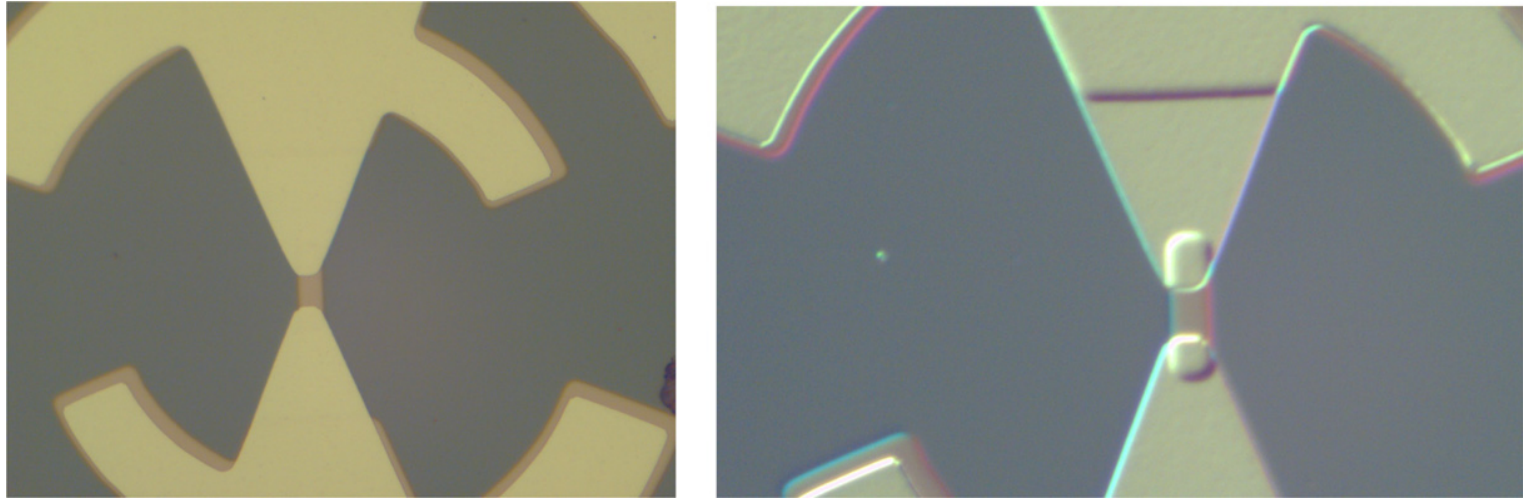

Figure 1. Optical image of a CEB with a log-periodic antenna: a plain photograph and a quasi-3D picture of the central part.

transition temperature. It has already been shown [1-3] that a CEB with electrothermal feedback can potentially give state of the art noise performance in the presence of typical background power loads seen in astronomical observations; e.g. from atmospheric loading and fluctuations. The time constant of the CEB can be considerably reduced by negative electrothermal feedback to the level of $10 \mathrm{~ns}$, which is several orders of magnitude shorter than that of TES detectors. The CEB is therefore a very promising device for future ground-based, balloon-borne and space telescopes due to its fast response, high sensitivity and high saturation. The fast response is absolutely crucial for fast scanning of large format bolometric interferometers that require the switching of several hundred baselines.

To optimize the performance, new technologies are being explored [3,4], including the employment of titanium-based CEB devices [4]. In a previous publication, we have reported the DC test results for Ti-based SIN tunnel junctions fabricated in direct-write technology [4] and demonstrated high performance $I V$ curves. The key feature of this technology is the use of a very thin and flat Ti absorber with no bending parts or steps, which is deposited prior to the tunnel junctions and the superconducting counter-electrode. In this paper we present the optical testing results for detectors fabricated using the same technique, with minor modifications in the patterning procedure and tunnel barrier thickness.

The chip layout of the devices presented here contains CEB devices integrated with a log-periodic antenna for measurements at frequencies between 50 and $500 \mathrm{GHz}$ (figure 1), and a double-dipole antenna that is optimized for measurements in the $300 \mathrm{GHz}$ frequency band (figure 2). Both dipole and log-periodic antennas are fabricated on a $\mathrm{Si}$ wafer, oxidized thermally to form a $400 \mathrm{~nm}$ thick $\mathrm{SiO}_{2}$ layer. There are 24 chips, $7 \mathrm{~mm} \times 7 \mathrm{~mm}$ each, on a single Si wafer.

\section{Fabrication}

The samples have been fabricated using photo-lithography using the technology of fabricating tunnel junctions proposed by Kuzmin [4]. This procedure uses ex situ Ti oxidation for the creation of the $\mathrm{TiO}_{2}$ insulating barrier. The process employs two steps of lithography and deposition: first the Ti absorber

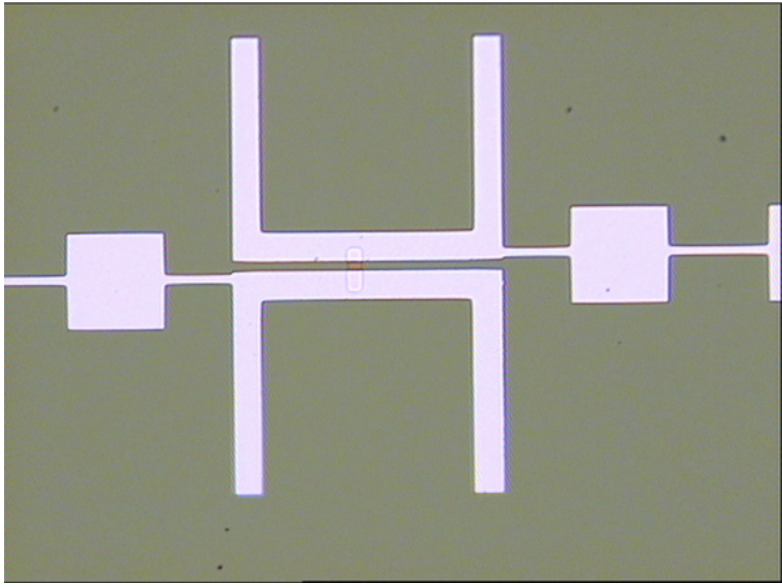

Figure 2. Photograph of a double-dipole antenna designed for $350 \mathrm{GHz}$, with CEB in the center.

is deposited and oxidized and then the $\mathrm{Al}$ counter-electrode is deposited and formed. In the first step, a photoresist is spun and baked on a thermally oxidized silicon substrate for the subsequent optical lithography. After exposure and development, a Ti film is deposited by magnetron sputtering. (Alternatively, the Ti absorber can be deposited and patterned using wet etching in $1 \% \mathrm{HF}$ solution.) After lift-off and oxygen plasma etching, the surface of $\mathrm{Ti}$ is cleaned using ion milling in an $\mathrm{Ar}$ ion beam and oxidized in an oven in air ambient. Then an $\mathrm{Al}$ film is deposited and patterned by lithography and chemical wet etching.

\section{Experiment results and analysis}

DC measurements of the devices with log-periodic antennas were performed at Oxford Physics using a HELIOX-AC-V cryostat manufactured by Oxford Instruments. DC testing was performed in current bias mode using a bias resistance of $20 \mathrm{M} \Omega$ to $2 \mathrm{G} \Omega$. Noise voltage spectra were measured using a room temperature MOSFET readout amplifier and a lock-in detector. Based on DC testing, a noise equivalent power (NEP) of NEP $\sim 10^{-17} \mathrm{~W} \mathrm{~Hz}^{-1 / 2}$ was estimated by measuring the output noise and dividing by a voltage to power response $\mathrm{d} V / \mathrm{d} P$ estimated from $\mathrm{d} V / \mathrm{d} T$ measurements. 


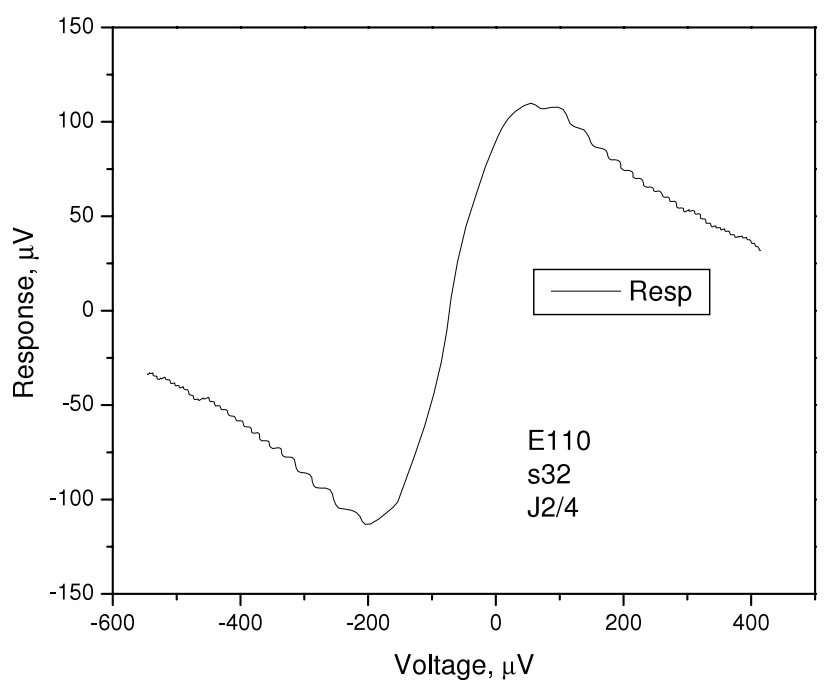

Figure 3. The optical response to a hot/cold load, with a source temperature difference of $3 \mathrm{~K}-270 \mathrm{mK}$.

Next, the optical response was measured by the hot/cold load method. For this a rotating stage with a $\mathrm{Cu}$ reflector and a blackbody surface inside the $3 \mathrm{~K}$ shield of the cryostat was used. Magnets placed on the stage allow an external magnet placed outside the cryostat to position either the absorber or reflecting surface in front of the bolometer. The detector chip was mounted behind a waveguide horn, used to concentrate the beam pattern of the detector's antenna on the reflector or blackbody source. By swapping the reflector and the blackbody source, we change the incident radiation temperature seen by the detector from $3 \mathrm{~K}$ to $270 \mathrm{mK}$ and measure the changes in the bias voltage at fixed tunnel current. The measured response for the detector integrated with a double-dipole antenna is $\Delta V_{\text {out }}=120 \mu \mathrm{V}$ (see figure 3).

The RF properties of a double-dipole antenna were studied before [5] and justified in our earlier works using the Hilbert-spectroscopy technique [6]. Based on published data and our previous experimental results, we estimate the bandwidth of our CEB-antenna IC to be around $100 \mathrm{GHz}$. Integrating Planck's law across the detector's RF bandwidth, the power received by a $300 \mathrm{GHz}$ antenna in a $100 \mathrm{GHz}$ bandwidth at a radiation source temperature of $3 \mathrm{~K}$ corresponds to $\Delta P=3.8 \times 10^{-13} \mathrm{~W}$. Combining this with the maximum measured voltage response of $\Delta V=$ $120 \mu \mathrm{V}$, this yields an estimate of the voltage-power response of $\mathrm{d} V / \mathrm{d} P=3.2 \times 10^{8} \mathrm{~V} \mathrm{~W}^{-1}$. In this calculation, we neglected the RF power irradiated at $270 \mathrm{mK}$ that is orders of magnitude lower than for $3 \mathrm{~K}$ due to an exponential temperature dependence. For a measured output noise of $V_{n}=10 \mathrm{nV} \mathrm{Hz}^{-1 / 2}$ the estimated optical NEP is NEP = $V_{n} /(\mathrm{d} V / \mathrm{d} P)=3.5 \times 10^{-17} \mathrm{~W} \mathrm{~Hz}^{-1 / 2}$.

In the next experiment, we employed calibrated measurements of the RF response of the CEB device with log-periodic antenna illuminated by a variable temperature blackbody source, mounted inside the cryostat. The CEB was tested in a $\mathrm{He}^{3}$ sorption cryostat with a base temperature of $280 \mathrm{mK}$. In order to select a frequency band of interest, a bandpass filter with a central frequency of about $300 \mathrm{GHz}$ and

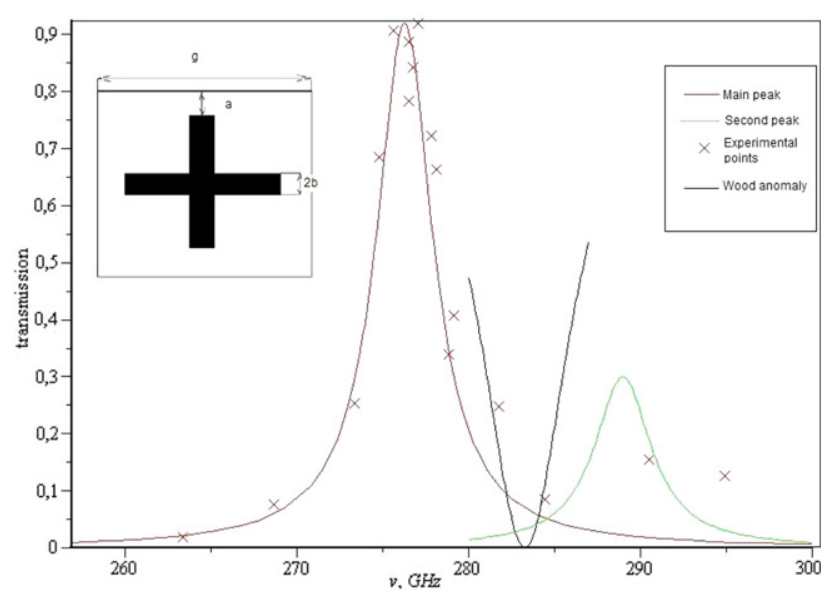

Figure 4. Measured characteristics of the mesh filter, experimental points and fitting curves, based on the Laurent approximation [9] for a quartz substrate. The main transmission peak corresponds to the resonance length of crosses. The central wavelength can be expressed by the formula [10] $\lambda_{0}=2 g-4 a-2 b$, where $2 a$ is the distance between the edges of the crosses, $g$ is the period of the grid, $2 b$ is the width of the cross, while the second peak appears due to the interference at the front and rear walls. A phenomenon called the Wood anomaly that is a transmission minimum due to interference along the substrate is observed at wavelengths around the grid pitch size $[10,11]$.

a bandwidth of $10 \mathrm{GHz}$ was placed between the RF source and the sample. The transmission coefficient of the mesh filter versus frequency measured using a backwards-wave oscillator is shown in figure 4.

DC $I V$ curves of the devices with log-periodic antennas were measured in a current bias mode; the optical response was measured by illuminating the devices with a blackbody source mounted inside the cryostat. The signal response (figure 5) was measured by comparing the $I V$ curves at different RF source temperatures. The voltage response to the incoming radiation at different emitter temperatures and bias currents is shown in figure 6 . The response up to $45 \mu \mathrm{V}$ was obtained for the voltage difference at the same bias current for 3 and $8 \mathrm{~K}$ blackbody source temperatures. Based on this value, the voltage to power response can be estimated using the Planck formula for given source temperatures.

Considering the CEB-antenna integrated circuit as a single-mode detector, we can easily calculate the radiation power $\delta P$ incident on the detector [7]. For a frequency range around the frequency of $f=300 \mathrm{GHz}$ with a bandwidth of $\delta f=10 \mathrm{GHz}$, the power emitted by a blackbody at a temperature of $T=8 \mathrm{~K}$ is given by

$$
\delta P=\frac{\kappa \cdot h f \cdot \delta f}{\exp \left(h f / k_{B} T\right)-1}=4.3 \times 10^{-12} \mathrm{~W},
$$

where $h$ is Planck's constant, $k_{B}$ is the Boltzmann constant, the emissivity of the blackbody source $\kappa=1$. A $20 \mathrm{~dB}$ attenuator with reflectivity of about $1 \%$ made of $\mathrm{NiCr}$ film on a CAPTON carrier was used as the blackbody source. It was glued onto a $\mathrm{Cu}$ plate equipped with a heater and a temperature sensor. The corresponding power for $3 \mathrm{~K}$ is $\sim 3 \times 10^{-13} \mathrm{~W}$. Subtracting the calculated power values at 


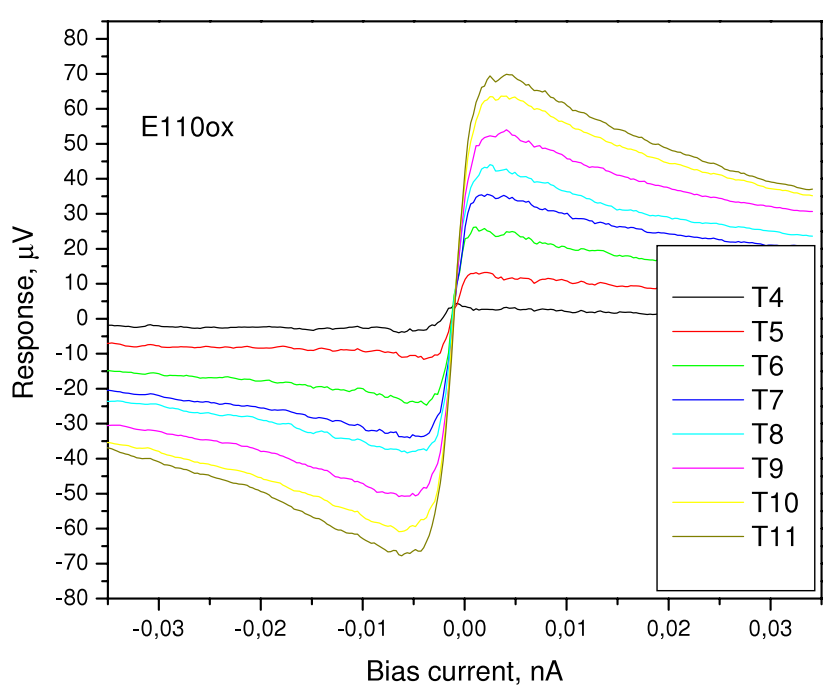

Figure 5. Optical response of a CEB versus bias current at different RF source temperatures; measured with a $300 \mathrm{GHz}$ bandpass filter. $T=4,5,6,7,8,9,10$ and $11 \mathrm{~K}$.

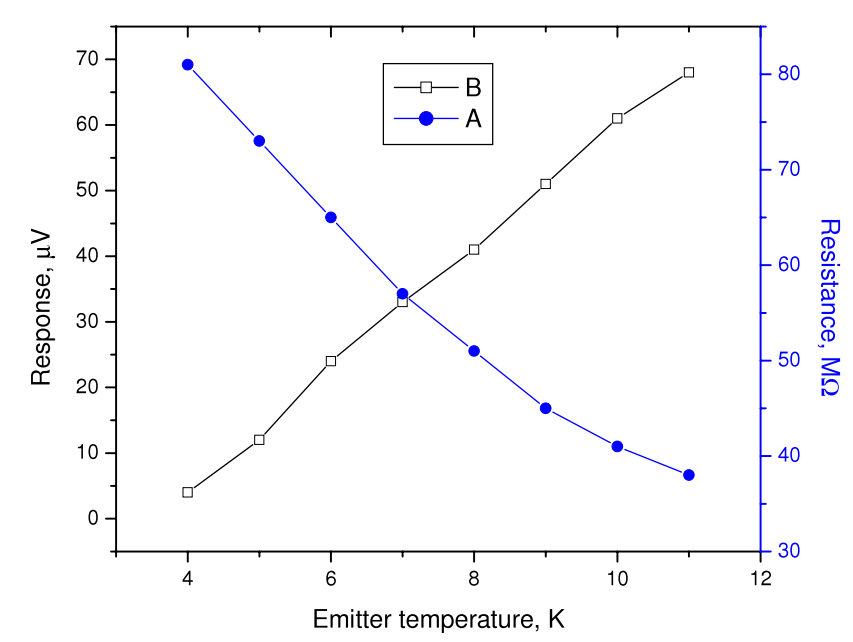

Figure 6. Dynamic resistance (A) and voltage response (B) versus emitter temperature.

source temperatures of 3 and $8 \mathrm{~K}$ yields $\Delta P=4 \times 10^{-12} \mathrm{~W}$. Taking a voltage change of $45 \mu \mathrm{V}$ for this power difference, we obtain:

$$
S=\mathrm{d} V / \mathrm{d} P \approx \Delta V / \Delta P=1.125 \times 10^{8} \mathrm{~V} \mathrm{~W}^{-1},
$$

assuming that the effective RF bandwidth is $10 \mathrm{GHz}$.

The optical NEP is obtained by dividing the total voltage noise of $V_{n}=10 \mathrm{nV} \mathrm{Hz}^{-1 / 2}$ by the voltage to power response:

$$
\mathrm{NEP}=V_{n} / S=8.88 \times 10^{-17} \mathrm{~W} \mathrm{~Hz}^{-1 / 2} .
$$

\section{Discussion}

The optical NEP value derived above is only an estimate. The connection from the RF source to the CEB detector contains several interfaces, and the losses due to the mismatches at different interfaces from the source to the detector in the present configuration are estimated at around $10 \mathrm{~dB}$. Optical losses at temperatures below that of the load will reduce the power received from the load by the detector, meaning that our value of $S$ is an underestimate. In turn, this suggests that our optical NEP is an overestimate of the intrinsic optical NEP of the device.

The voltage noise of $\sim 10 \mathrm{nV} \mathrm{Hz} z^{-1 / 2}$ was measured using the room-temperature amplifier box. As a result, the noise coming from the amplifier dominates the total noise measured.

Regarding fabrication, a straightforward and robust technology was chosen for the production of these devices. This was a reasonable choice for developing this experiment in that it allowed us to demonstrate the full functionality of the CEB device. It also allowed us to demonstrate the CEB-antenna integrated circuit as a feasible design.

However, our fabrication methods were not optimized in terms of device performance. The volume of the absorber was not chosen for best responsivity. Therefore, we expect improvements in the fabrication and design of this device and its bias circuit to yield improved responsivity and noise performance. With a reduced volume of absorber and in the voltage bias mode with SQUID readout, the CEB can achieve promising dark NEP levels [8].

For most practical cases the NEP will be determined by a background power load that is about $P_{b g}=5 \mathrm{pW}$ for ground-based observations at a signal frequency of $100 \mathrm{GHz}$, which corresponds to a photon NEP of

$$
\begin{aligned}
\mathrm{NEP}_{\text {phot }} & =\sqrt{2 P_{p b} E_{\text {quant }}}=\sqrt{2 P_{b g} h f} \\
& =2.4 \times 10^{-17} \mathrm{~W} / \sqrt{\mathrm{Hz}} .
\end{aligned}
$$

\section{Conclusions}

The results of DC and RF measurements on a cold-electron bolometer deposited across a double-dipole antenna and a log-periodic antenna on a planar $\mathrm{Si}$ substrate have been presented. The devices have been fabricated using direct-writing, with the absorber deposited prior to the superconducting counter-electrodes. The detector could be a potential candidate for the next generation of space cosmology missions and is also of interest to ground-based astronomical experiments as a result of the simplicity of its integration with planar circuit technology, high saturation power and fast response. For the samples measured without filters, the estimated noise equivalent power in the current biased mode and read by room temperature op-amp electronics was $\mathrm{NEP}=3.5 \times 10^{-17} \mathrm{~W} \mathrm{~Hz}^{-1 / 2}$.

\section{Acknowledgments}

This work was supported in part by a Wykeham Scholarship from New College, Oxford, the Physics Department of Oxford University, the Swedish Institute, the Swedish Vetenskapsrådet and the Russian Ministry of Sciences and Education under grant No. 11.G34.31.0029. 


\section{References}

[1] Kuzmin L 2000 On the concept of a hot-electron microbolometer with capacitive coupling to the antenna Physica B 284-288 2129

[2] Kuzmin L 2001 Optimization of the hot-electron bolometer for space astronomy SNED Proc. (Naples) pp 145-54

Kuzmin L and Golubev D 2002 Physica C 372-376 378

[3] Kuzmin L, Yassin G, Withington S and Grimes P 2007 An antenna coupled cold-electron bolometer for high performance cosmology instruments Proc. 18th ISSTT (Pasadena, March 2007) pp 93-9

[4] Otto E, Tarasov M and Kuzmin L 2007 Ti-TiO2-Al normal metal-insulator-superconductor tunnel junctions fabricated in direct-write technology Supercond. Sci. Technol. 20 865-9

[5] Van der Vorst M J M 1999 Integrated lens antennas for submillimetre-wave applications $P h D$ Thesis Technische Universiteit Eindhoven
[6] Kosarev E L, Shul'man A Ya, Tarasov M A and Lindström T 2003 Deconvolution problems and superresolution in Hilbert-transform spectroscopy based on a.c. Josephson effect Comput. Phys. Commun. 151 171-86

[7] Karasik B and Cantor R 2011 Demonstration of high optical sensitivity in far-infrared hot-electron bolometer Appl. Phys. Lett. 98193503

[8] Kuzmin L 2008 Array of cold-electron bolometers with SIN tunnel junctions for cosmology experiments J. Phys.: Conf. Ser. (JPCS) 97012310

[9] MacDonald M E and Grossman E N 1999 Far-infrared band-pass filters SPIE 2842377

[10] Möller K D, Farmer K R, Ivanov D V P, Sternberg O, Stewart K P and Lalanne P 1999 Thin and thick cross shaped metal grids Infrared Physics and Technology vol 40 (New York: Elsevier) pp 475-85 doi:10.1016/ S1350-4495(99)00031-6

[11] Winnewisser C, Lewen F T, Schall M, Walther M and Helm H 2000 Characterization and application of dichroic filters in the 0.1-3-THz region IEEE Trans. Microwave Theory Techniques 48 (4) 744-9 